\title{
The effects of mixing time on cement paste slurry transport and mechanical property
}

\author{
Liuhua Yang University of Science and Technology Beijing, China \\ Hongjiang Wang University of Science and Technology Beijing, China \\ Shunchuan Wu University of Science and Technology Beijing, China \\ Aixiang Wu University of Science and Technology Beijing, China \\ Yong Wang University of Science and Technology Beijing, China \\ Xu Zhou University of Science and Technology Beijing, China \\ Lianfu Zhang University of Science and Technology Beijing, China \\ Xixiang Yang Jiashi Tonghui Copper Mine Co., Ltd, China
}

\begin{abstract}
The optimization of mixing time could benefit transport and mechanical performances of paste due to the enhancement of cement dispersion and paste fluidity induced by mixing process. However, research on the effects of mixing time during paste preparation has rarely focused on the reasons and mechanisms for the changes in characteristic properties. In this paper, a mining paste mixing system was simulated based on the similarity principle that recorded the slump and mechanism of paste among several mixing modes and on the clarify principles with which mixing process affected paste's properties. The results indicated that in consideration of equal linear velocity, the transport and mechanical properties of paste were definitely improved with time when mixing time was less than 3 min. Once mixing time exceeded 3 min, the properties of paste were improved at a slower rate until they became insensitive to mixing action at four minutes. Taking into account the paste properties, operation efficiency and energy consumption, the optimal mixing time was determined as approximately $3 \mathrm{~min}$. The reform towards mixing system which met paste filling flow of 90 $\mathrm{m}^{3} / \mathrm{h}$ was implemented by extending mixing time to $3.33 \mathrm{~min}$. Consequently, the slump was definitely enhanced by $\leq 10 \%$. Besides, the uniaxial compression strength (UCS) of specimens curing for $28 d$ was enhanced from 0.69 to $1.37 \mathrm{MPa}$.
\end{abstract}

\section{Introduction}

During paste preparation, effects of certain phenomena on fluidity and hardening characteristics of cementbased grouts have been reported (Takahashi et al., 2011). Deterioration of fluidity and hardening will affect the paste performance and workmanship quality, hence resulting in subsequent structural defects. In cemented filling mining industry, mixing process is the key to ensuring the desired quality and controlled properties of the materials. Therefore, improper mixing lists as one of the most serious concerns of mining engineers (Juez et al., 2017) and has attracted increasing attention in recent decades due to the development of new materials with more requirements for mixing time (Cazacliu et al., 2009), such as the unclassified tailing paste which strictly requires a strong awareness of environmental protection (Henriquez et al., 2009). 
To ensure good quality and high productivity, engineers tend to prioritize the mixing time with an attempt to minimize it to the greatest degree (Ferraris, 2001). Similar research could be found in other industries related with cementitious materials (Lombois-Burger et al., 2006; Pileggi et al., 2001; Brand et al., 2015; Wendling et al., 2016). The present research describes and assesses the effects of several mixing parameters on the properties of paste materials, such as their fluidity and hardening characteristics.

Unclassified tailing paste is a granular fluid, i.e. a complex mixture of unclassified tailing, crushed aggregate, powders, water and chemical admixtures (Wang et al., 2008; Yang et al., 2016). Its mixing mainly consists of dispersing constituents into the mixed volume and then having the mixture evolve from a wet granular state to a granular suspension microstructure (Cazacliu, 2008; Doucet et al., 2008). While some of the research concerning the dispersion of constituents in concrete or cement paste has been conducted (Wang et al., 2005; Board, 1997; Chopin et al., 2007; Jézéquel et al., 2007; Vandanjon et al., 2003; Beitzel et al., 2003), less interest has been given to the unclassified tailing paste mixing and the study of the homogenization process is still on the macroscopic scale (Lindenberg et al., 2008). In a sedimentary copper mine, with the tailings containing a considerable amount of clay, the yield stress and viscosity of the preparing paste are large and the cement particles are difficult to disperse. Ultimately, these characteristics result in poor paste fluidity and uneven backfilling body strength distribution. In order to solve the uneven dispersion of cement, this paper analyzes the factors of materials movement and homogenization in the mixing process, and proposes measures to improve the homogenization of high clay and viscosity paste.

\section{Problems of the paste mixing system}

High clay tailings with an argillaceous content of more than $60 \%$ feature high viscosity, large yield stress, low dispersion and slow coagulation performance. Those characteristics seriously affect the homogenization of the slurry, hence resulting in high cement consumption, low production efficiency, high backfilling costs, doubled resistance to transmission and resistance to flattening under gravity in the stope. In this mine, the whole tailings and coarse aggregate (particle size $\leq 10 \mathrm{~mm}$ ) with the cement as a binder produce backfill paste when adding a pumping agent to reduce the transmission resistance and improve the solid concentration with the same fluidity. The mixture of coarse aggregate, cement, pumping agent and thick tailings is crucial for the transport and mechanical properties of paste and triggers a high demand for the technique and equipment of paste mixing.

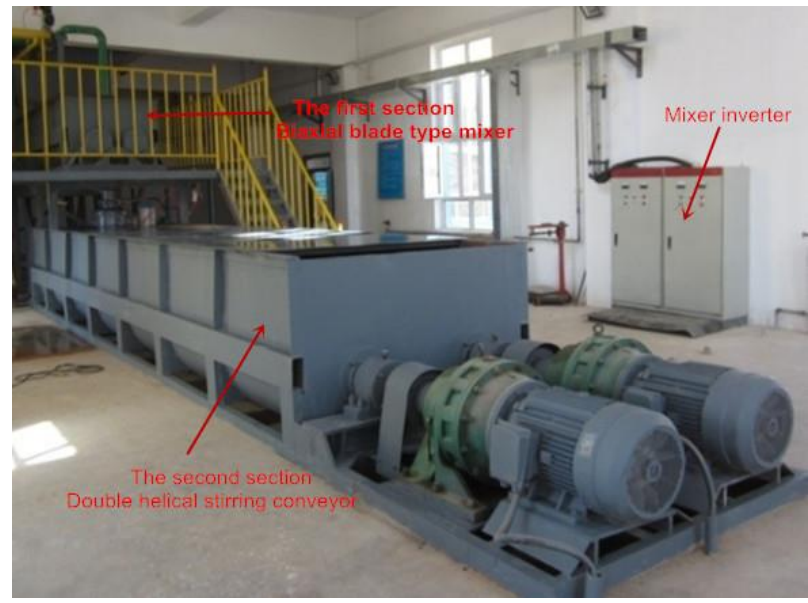

(a)

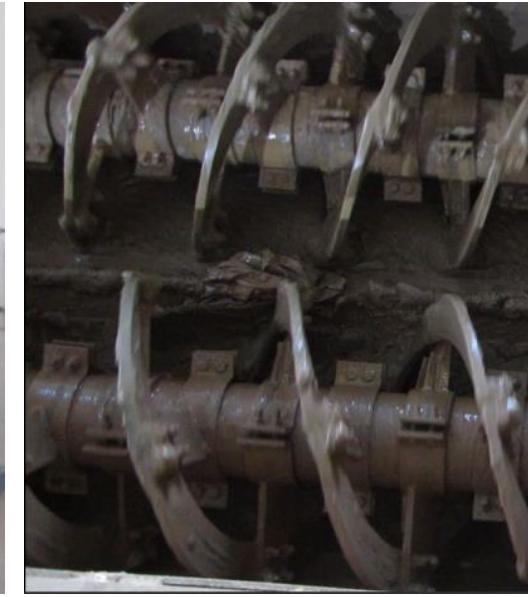

(b)

Figure 1 The paste mixing system; (a) the paste mixer appearance; (b) blades of mixer

The mixing system has two continuous stirring parts, wherein the first section is a biaxial blade type mixer and the second section a double helical stirring conveyor (see Figure 1). The components of paste are initially 
mixed in the first section to quickly disperse and moisten the material. The slurry then moves uniaxially from inlet to outlet in the second section. The short resistance time and poor mixing effect together induce low slurry fluidity for relatively poor dispersion and hydration of cement. The improvement mainly enhances the mixing capacity of the two-stage double-screw mixing system to solve relevant problems.

\section{Experimental method}

\subsection{Experimental program}

Generally, movement and collision induced by particles in mixers should be forceful enough to improve the homogeneity of paste slurry with paste materials mixed both in micro- and macro-sizes and within certain time limit. The improvement of the mixer's rotation speed $r$ is key to obtaining even paste both at micro- and macro-levels. However, it is proved that mixers cannot perform well once the mixing speed exceeds $40 \mathrm{r} / \mathrm{min}$ (linear velocity of $1.46 \mathrm{~m} / \mathrm{s}$ ). To simulate the performances of practical mixing systems, the similarity principle was applied to emulate paste mixing process with the same linear velocity $(1.46 \mathrm{~m} / \mathrm{s})$. Transport and mechanical properties with different mixing time were examined for the identification of the optimal mixing time in paste preparation. The experiment was implemented based on single variable design described in Table 1.

Table 1 Experiment design of mixing time optimization

\begin{tabular}{ccccc}
\hline Mixing time (min) & Tail waste ratio* & Cement-sand ratio & Mass fraction (\%) & Dosage of addition (\%) \\
\hline 1 & 4 & $1: 6$ & 76 & 2 \\
2 & 4 & $1: 6$ & 76 & 2 \\
3 & 4 & $1: 6$ & 76 & 2 \\
4 & 4 & $1: 6$ & 76 & 2 \\
5 & 4 & $1: 6$ & 76 & 2 \\
\hline
\end{tabular}

*Note: Tail waste ratio means the radio of tailings and crushed aggregate.

\subsection{Results}

Six samples were obtained from each group of the slump and the uniaxial compression strength (UCS) of paste and were measured respectively after curing. The results were averaged and are presented in Figures 2 and 3.

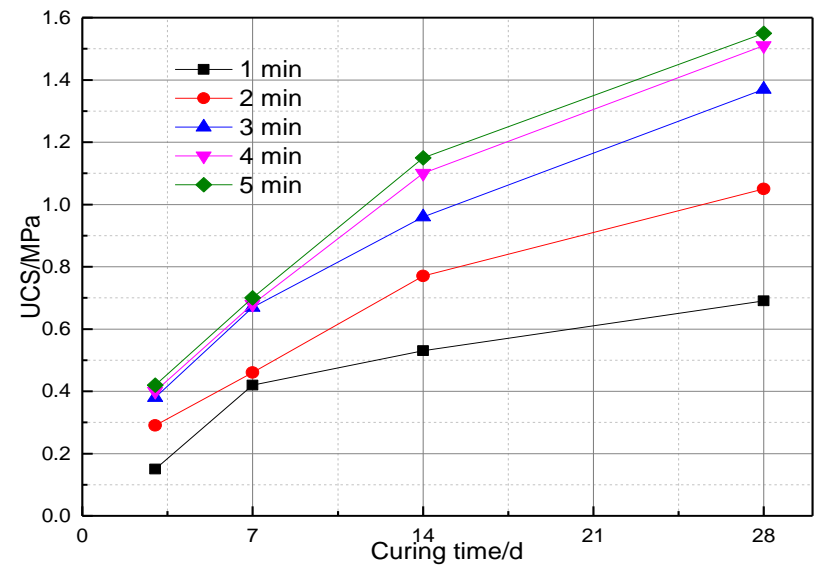

Figure 2 Experimental results of UCS 


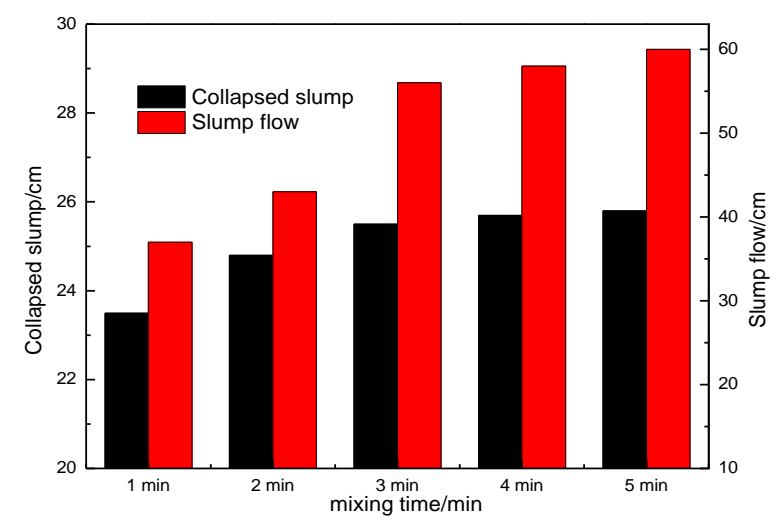

Figure 3 The paste fluidity obtained in different mixing time

When mixing time is less than $3 \mathrm{~min}$, the transport and mechanical properties of paste prepared in mixers improved during the process of mixing. However, the improvement of slump, dispersion and UCS is slower between 3 to $4 \mathrm{~min}$. The properties almost remain unchanged when mixing time exceeds $4 \mathrm{~min}$. A low degree of UCS dominating in all samples with a mixing time of less than $2 \mathrm{~min}$ and the mean of UCS changes within a wide range. The increase of mixing time leads to the decrease of the deviation of UCS tests. It also shows that low UCS and poor fluidity of paste is predominantly caused by uneven mixing.

\section{$4 \quad$ Industrial field test}

Due to problems in paste mixing system, both volumetric efficiency $\lambda$ and mixing speed can be enhanced by mixing optimization experiments. Consequently, the poor dispersion of cement, low fluidity of slurry, and low strength of paste together were solved for extended mixing time and strengthened mixing effect.

Prior to the modification: without the water level controller, the volumetric efficiency $\lambda$ of stirred tank was rather low and the mixing time never exceeded $1 \mathrm{~min}$. In addition, the performance of material dispersion was poor for mixing speed of $30 \mathrm{r} / \mathrm{min}$.

After the modification: the use of water level controller improved the mixer volumetric efficiency of stirred tank and extended the mixing time. Besides, the use of mixer inverter increased mixing speed from 30 to $40 \mathrm{r} / \mathrm{min}$ while reducing the splash from mixer. In addition, the mixer inverter simultaneously adjusted relative motion of axis so that the modes by which slurry movement was controlled switched much easier between single-cycle and multi-cycle. In multi-cycle mode, a closed bigger cycle was formed along the axis under biaxial interaction.

To verify the performance of mixing system after modification, a homogenized paste mixing industrial test was carried out in the field. The percentage of the paste with cement-sand ratio of 1:6, tail-waste ratio of 4:1 and pumping admixture ( $2 \mathrm{wt} \%$ of cement content) was $76 \%$. The samples should be extracted from outlet of stirred tank after the system had steadily operated. The slump and divergence of the slurry samples were measured immediately. Having divided into four groups, the slurry was molded and curried for 3, 7, 14 and $28 \mathrm{~d}$, respectively. Analyze influences to paste mechanical properties induced by different mixing modes and results are shown in Table 2. Besides, the first group carried out before the reform was designed as control group. 
Table 2 Parameters of field industrial test

\begin{tabular}{|c|c|c|c|c|c|c|c|c|}
\hline \multirow[b]{2}{*}{ Stirring method } & \multirow{2}{*}{$\begin{array}{l}\text { Mixing time } \\
\text { (min) }\end{array}$} & \multirow{2}{*}{$\begin{array}{l}\text { Mixing speed } \\
\text { (r/min) }\end{array}$} & \multicolumn{4}{|c|}{ UCS (MPa) } & \multicolumn{2}{|c|}{ Fluidity (cm) } \\
\hline & & & $3 d$ & $7 d$ & $14 \mathrm{~d}$ & $28 d$ & Slump & $\begin{array}{c}\text { Slump } \\
\text { flow }\end{array}$ \\
\hline $\begin{array}{c}\text { Prior to } \\
\text { modification }\end{array}$ & 0.57 & 30 & 0.15 & 0.42 & 0.53 & 0.69 & 23.5 & 37.0 \\
\hline After modification & 3.33 & 40 & 0.38 & 0.67 & 0.96 & 1.37 & 25.5 & 56.0 \\
\hline
\end{tabular}

As the field industrial test shows, the modified system enhanced both the slump and strength of paste and solved the problem of poor dispersion of cement in high-mud unclassified tailing paste mixing process. Under strong mixing action in particular, the thixotropic activation occurred in paste slurry and the transport and mechanical properties improved acutely. The solid dispersion of paste tailings in slurry was liquefied and formed the colloid which resembled the non-Newtonian fluid with good fluidity and high evenness. With the same cement-sand ratio of 1:6 and tail-waste ratio of 1:3, the slump and slump flow of paste increased by 8.5 and $51.4 \%$, respectively. In addition, the strength of specimen curing for $28 \mathrm{~d}$ improved from 0.69 to $1.37 \mathrm{MPa}$.

\section{The analysis of mixing homogenization mechanism of paste}

To analyze how the microstructure of particles changes in paste mixing process, comparison of particle movement between pre- and post-modification as well as the calculation of mixing time in both formations were implemented.

\subsection{The mixing time before and after modification}

The principle of forced mixer highlights the importance of increasing the collision and friction among the mixed particles to well distribute the materials. Various mixing time should be applied to different types of mixing. Compared with liquid, paste slurry has larger yield stress and lower fluidity. Therefore, mixing paste requires longer mixing time and more intensive energy.

\subsubsection{Before modification}

Prior to modification, materials were driven by blades from the inlet to outlet to finish a mixing cycle within a certain time that is defined as the passing time of materials. According to preceding characteristics, the computational model of mixing time under convection arrangement was deduced.

$$
\mathrm{T}=L /(S \cdot N)
$$

Where:

$$
\begin{array}{ll}
\mathrm{T} & =\text { mixing time, } \mathrm{min} ; \\
S & =\text { thread pitch, } \mathrm{m} ; \\
N & =\text { speed of spindle, } \mathrm{r} / \mathrm{min} ; \\
L & =\text { length of mixer, } \mathrm{m} ;
\end{array}
$$

Provided that the speed is $30 \mathrm{r} / \mathrm{min}$, the thread pitch is $0.4 \mathrm{~m}$ and the length of mixer is $6.84 \mathrm{~m}$, the mixing time calculated by Equation (1) is $0.57 \mathrm{~min}$.

From the above equation, it can be seen that before modification, the mixing time is relative to the parameters of thread pitch, the speed of spindle and the length of mixer, among which only the speed of spindle could be adjusted. According to the equation, the mixing time is inversely proportional to the speed of spindle. When the speed is approximately zero, the mixing time tends to be infinite. However, when the speed is zero, there is no agitation and the production efficiency is zero, such as the situation where the paste 
gets stacked in the mixer with blades being stable. Therefore, the relationship between agitation and mixing time is similar to that between Spear and Shield and hence, cannot be optimized. Besides, the paste slurry can easily splash out during the mixing process due to the low liquid level and the exposure of certain parts of blades to air.

\subsubsection{After modification}

To improve the mixer performance, measures should be taken to effectively adjust the liquid level. By analyzing the motional pattern of materials in the modified mixer, it can be found that the materials could be multi-cyclically stirred and the mixing time be extended. As a result, the computational model is obtained as follows:

$$
\mathrm{t}=60 \lambda \frac{Q}{q}
$$

Where:

$\mathrm{t} \quad=$ mixing time, $\min$;

$\lambda=$ volumetric efficiency, which is related to liquid level, $0 \leq \lambda \leq 1$;

$Q \quad=$ effective volume of the mixer, $\mathrm{m}^{3}$;

$q \quad=$ flux of backfill, $\mathrm{m}^{3} / \mathrm{h}$;

Provided that $Q$ is $5 \mathrm{~m}^{3}, \lambda$ is 1 , and $q$ is $90 \mathrm{~m}^{3}$, the mixing time calculated by Equation (2) is $3.33 \mathrm{~min}$. Under this condition, materials can be homogenized and fluidized. From Equation (2), factors influencing the mixing time after modification include the volumetric efficiency and the effective volume of the mixer and flux and exclude the mixing velocity.

Therefore, mixing time can be extended by controlling the liquid level and optimizing the flux of backfill. Meanwhile, improving the speed of stirring can also improve the effect of mixing, which realizes the unification of the speed and effect of mixing.

\subsection{Main microstructural evolution taking place during paste mixing}

Taking into account the changing physical state of tailings, coarse aggregates and cement during the mixing in-site, the research tracks the materials' fluidization process and presents in Figure 4 the change of microstructure in tailings paste mixing. In the figure, the horizontal axis denotes the mixing process, the vertical axis the change of the materials' volume, the positive direction the aggregation of particles, and the negative direction the dispersion of particles. 


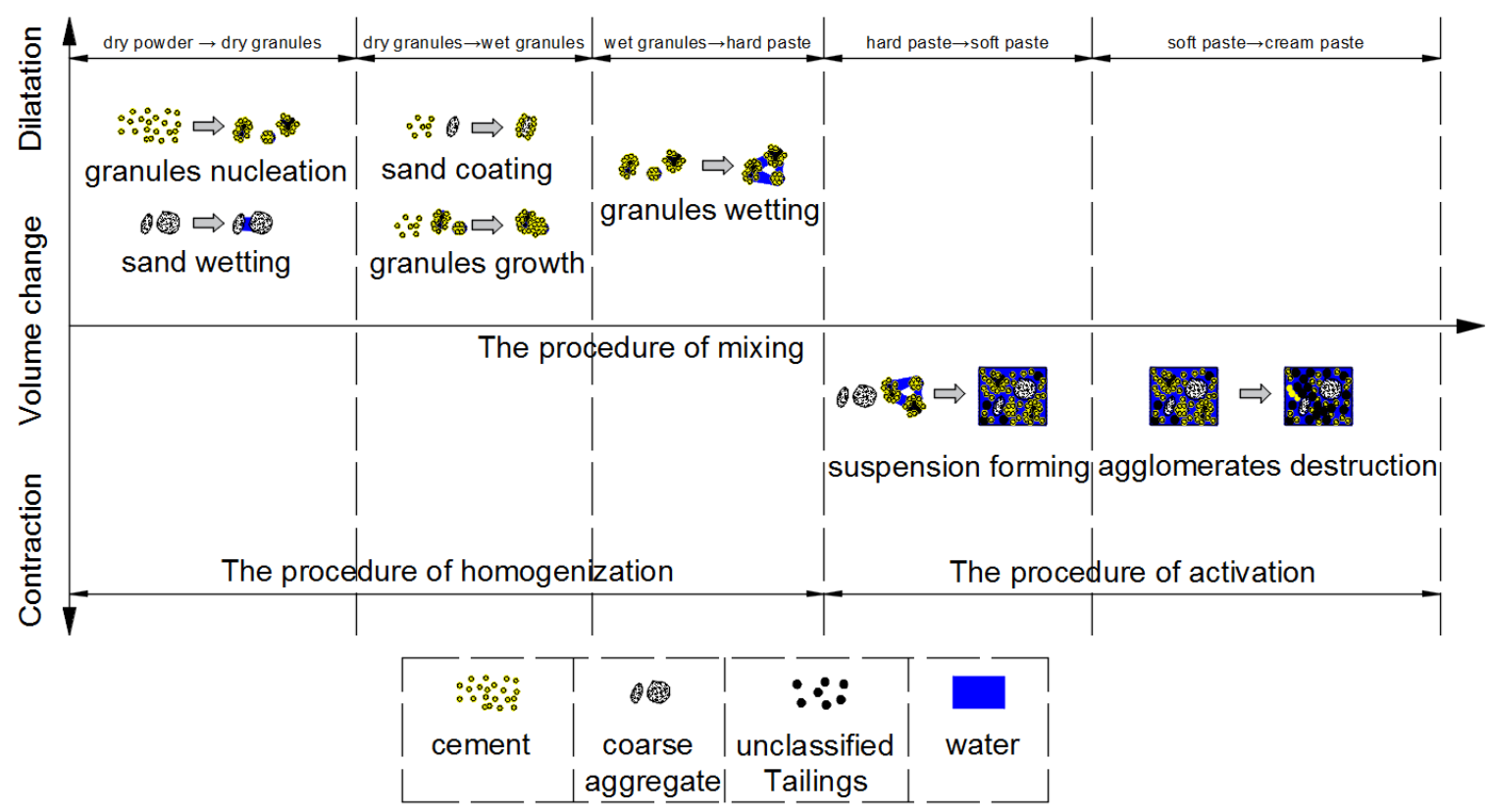

Figure 4 Main types of microstructural change involved in tailings paste mixing

When cement and coarse aggregates are mixed into unclassified tailings, cements and tailings generate kernel-shaped particles under the tension of water, and coarse aggregates get dampened first because they are not easily gathered. Under the perturbation of blades, wet aggregates contact with dry-powder cement, making the aggregates surface encased by cement. A portion of kernel-shaped aggregates become larger after absorbing a small amount of water and contacting with dry cement particles. In the process of mixing, the surface of gathered cements, tailings and coarse aggregates get dampened and attracted to one other by the effect of liquid bridge. Through forceful stirring, the liquid bridges between gathered particles are broken and the mixtures become suspensions. Under strong shear, the flocs in the suspensions are sufficiently broken and become activated paste, accomplishing the transformation from materials to paste. By multi-cyclically strong agitate, the shear and impact between materials and blades are enhanced and thus could stimulate the collision among materials, promote the dispersion of materials, and accelerate the slurry homogenization in microcosmic and activate paste. The advisable way of mixing paste is to make materials generate more trajectory intersection, to extend the distance and time of stirring, to promote the cycle of materials and accelerate the homogenization at macroscopic and microcosmic scales.

\section{Conclusions}

High-mud unclassified tailings slurry was characterized by high viscosity, high yield stress and poor dispersion of cement mass. Unmodified paste mixing system induced poor performance in materials mixing, cement hydration, slurry transport and mechanical properties. The strength of paste specimen curing for $28 \mathrm{~d}$ with cement-sand ratio of 1:6 and tail-waste ratio of 1:3 is $0.69 \mathrm{MPa}$.

The optimization of paste mixing modes, extending mixing time from $0.57 \mathrm{~min}$ to $3.33 \mathrm{~min}$ and increasing mixing speed with the filling flow speed of $90 \mathrm{~m}^{3} / \mathrm{h}$, enhanced the mixing effect by accelerating the process of homogenization and fluidization of the paste materials. The experiment shows the slump and divergence of paste increased by 8.5 and $51.4 \%$, respectively. In addition, the UCS of specimens curing for $28 \mathrm{~d}$ was strengthened to $1.37 \mathrm{MPa}$.

The optimal mixing time of practical mining paste was proposed as $3 \mathrm{~min}$ after the simulation of practical mixing system. Then field industrial test was implemented to verify the rationality of optimal mixing time by comparing the mixing effect and microstructural change of paste materials prior to and after modification. 
The industrial test indicated that poor dispersion of cement in high-mud unclassified tailing paste could be well solved through field modification.

\section{Acknowledgements}

This work is financially supported by the National Twelfth Five-Year Science and Technology Support Program (2012BAB08B02), the National Natural Science Foundation of China (51374034), and the University of Science and Technology Beijing.

\section{References}

Beitzel, H., Charonnat, Y. and Beitzel, M. 2003, 'Assessment and classification of performance mixers', Materials and Structures, vol. 36 , no. 258 , pp. $250-264$.

Brand, A.S., Roesler, J.R. and Salas, A. 2015, 'Initial moisture and mixing effects on higher quality recycled coarse aggregate concrete', Construction and Building Materials, vol. 79, pp. 83-89.

Cazacliu, B. 2008, 'In-mixer measurements for describing mixture evolution during concrete mixing', Chemical Engineering Research and Design, vol. 86, no. 12, pp. 1423-1433.

Cazacliu, B. and Roquet, N. 2009, 'Concrete mixing kinetics by means of power measurement', Cement and Concrete Research, vol. 39, no. 3, pp. 182-194.

Chopin, D., Cazacliu, B., Larrard, F.D. and Schell, R. 2007, 'Monitoring of concrete homogenization with the power consumption curve', Materials and Structures, vol. 40, no. 9, pp. 897-907.

Doucet, J., Bertrand, F. and Chaouki, J. 2008, 'A measure of mixing from Lagrangian tracking and its application to granular and fluid flow systems', Chemical Engineering Research and Design, vol. 86, no. 12, pp. 1313-1321.

Ferraris, C.F. 2001. 'Concrete mixing methods and concrete mixers: State of the art', Journal of Research of the National Institute of Standards and Technology, vol. 106, no. 2, pp. 391-399.

Lindenberg, C., Schöll, J., Vicum, L., Mazzotti, M. and Brozio, J. 2008. 'Experimental characterization and multi-scale modeling of mixing in static mixers', Chemical Engineering Science, vol. 63, no. 16, pp. 4135-4149.

Lombois-Burger, H., Colombet, P., Halary, J.L. and Damme, V.H. 2006, 'Kneading and extrusion of dense polymer-cement pastes', Cement and Concrete Research, vol. 36, pp. 2086-2097.

Wendling, A., Mar, D., Wischmeier, N., Anderson, D. and Mclff, T. 2016, 'Combination of modified mixing technique and low frequency ultrasound to control the elution profile of vancomycin-loaded acrylic bone cement', Bone and Joint Research, vol. 5, pp. 26-32. 